\title{
Vector Control Algorithm for Electric Vehicle AC Induction Motor Based on Improved Variable Gain PID Controller
}

\author{
Gang Qin, ${ }^{1}$ Mushuang Liu, ${ }^{2}$ Jianxiao Zou, ${ }^{1}$ and Xiaoshuai Xin ${ }^{1}$ \\ ${ }^{1}$ School of Automation Engineering, University of Electronic Science and Technology of China, Chengdu 610023, China \\ ${ }^{2}$ School of Microelectronics and Solid-State Electronics, University of Electronic Science and Technology of China, \\ Chengdu 610023, China
}

Correspondence should be addressed to Mushuang Liu; lxwgws@163.com

Received 3 December 2014; Revised 28 December 2014; Accepted 28 December 2014

Academic Editor: Hui Zhang

Copyright (C) 2015 Gang Qin et al. This is an open access article distributed under the Creative Commons Attribution License, which permits unrestricted use, distribution, and reproduction in any medium, provided the original work is properly cited.

\begin{abstract}
The acceleration performance of EV, which affects a lot of performances of EV such as start-up, overtaking, driving safety, and ride comfort, has become increasingly popular in recent researches. An improved variable gain PID control algorithm to improve the acceleration performance is proposed in this paper. The results of simulation with Matlab/Simulink demonstrate the effectiveness of the proposed algorithm through the control performance of motor velocity, motor torque, and three-phase current of motor. Moreover, it is investigated that the proposed controller is valid by comparison with the other PID controllers. Furthermore, the AC induction motor experiment set is constructed to verify the effect of proposed controller.
\end{abstract}

\section{Introduction}

With the increased emphasis on saving energy and reducing emission, electric vehicles (EVs) have emerged as very strong candidates to achieve these goals [1-5]. Moreover, the acceleration performance of $\mathrm{EV}$, which affects a lot of performances of EV such as start ability, passing ability, driving safety, and ride comfort, is the key point of $\mathrm{EV}$ researches.

Vector control algorithm, which can accurately control the torque and has a wide control range of motor velocity and also has a current loop which can be used for current limiting protection, is widely used in EV driving control. However, the velocity loop controller of vector control algorithm, which uses traditional PID control algorithm generally, limits the dynamic performance of driving system and the acceleration performance of EV. During the last few years, the velocity loop controller of EV AC induction motor (ACIM) controller system is researched and improved unceasingly and many methods are presented. One method is using the fuzzy controller to replace velocity loop traditional PID controller and current loop traditional PID controller of vector control algorithm [6-8], which can make the control system track the different given velocity rapidly and without overshoot in different load and has strong ability against load disturbance, but its steady-state accuracy is not high because of no existing integration element. Another method is using the neutral network PID controller to replace velocity loop traditional PID controller of vector control algorithm [9-11], which has the advantages of adjusting velocity rapidly, zero overshoot, smooth and small-fluctuation control signals, and good system tracking, but it reduces the EV control performance due to learning slowly in learning process and long response time. Literature [12] also presented a method using the fuzzyPI controller which executes fuzzy control algorithm when velocity deviation is greater than given threshold and executes traditional PID control algorithm when velocity deviation is less instead of velocity loop traditional PID controller [13]. The method can make velocity response rapidly with small overshoot [14], but it is difficult to achieve completely smooth switching and may cause velocity hop when control algorithm switches, thereby affecting the driving safety and ride comfort when EV accelerates.

In this paper, we design a vector control algorithm for vehicle asynchronous motor based on improved variable gain PID controller which can make motor velocity rise rapidly and no overshoot. Moreover, it can satisfy the demands of 
EV driving system dynamic performance and acceleration performance no matter whether the EV runs in low velocity, normal velocity, high velocity, or variable velocity.

The sections are organized as follows. In Section 2, asynchronous motor model is studied. In Section 3, improved variable gain PID control algorithm is designed to improve the acceleration performance. In Section 4, vector control algorithm for EV asynchronous motor based on improved variable gain PID controller is proposed. Furthermore the stability condition is given. In Section 5, the effectiveness of controller is demonstrated by simulation with Matlab/Simulink (Figure 4). Section 6 presents some concluding remarks.

\section{AC Induction Motor Model}

ACIM is widely applied to EV driving system, which has many good characteristics such as robustness, durability, simple structure, reliable operation, low cost, low torque ripple, low noise, no position sensor, and high velocity limit. The design of ACIM for EV which is different from normal ACIM and must satisfy the power performance of EV must have the following characteristics: (1) constant power output and big velocity adjustable range for satisfying the demands for flat road, overtaking, and so on when run in high/low velocity, (2) smaller mass and volume in the condition of certain power level, and (3) robust structure and resistance to vibrations [15].

This paper which takes ACIM for example researches motor mathematical model of EV driving system. The mathematical model of ACIM is a nonlinear, high order, close coupling multivariable system. Ignore these factors such as core loss, space harmonics, the change of frequency, the change of temperature, and the saturation of magnetic circuit on the impact of winding resistances when establishing motor model [16].

A physical model of ACIM is shown in Figure 1. The three-phase winding resistances which are $120^{\circ}$ phase different in the space are symmetrical, and the mutual inductance and self-inductance of every winding resistance are constant. The mathematical models of ACIM, which consist of voltage matrix equation, magnetic linkage matrix equation, and torque equation, can be obtained according to the physical model of ACIM.

Based on the voltage balance principle of three-phase stator winding resistances, the voltage matrix equation [17] can be written as

$$
\left[\begin{array}{l}
u_{A} \\
u_{B} \\
u_{C} \\
u_{a} \\
u_{b} \\
u_{c}
\end{array}\right]=\left[\begin{array}{cccccc}
R_{s} & 0 & 0 & 0 & 0 & 0 \\
0 & R_{s} & 0 & 0 & 0 & 0 \\
0 & 0 & R_{s} & 0 & 0 & 0 \\
0 & 0 & 0 & R_{r} & 0 & 0 \\
0 & 0 & 0 & 0 & R_{r} & 0 \\
0 & 0 & 0 & 0 & 0 & R_{r}
\end{array}\right]\left[\begin{array}{c}
i_{A} \\
i_{B} \\
i_{C} \\
i_{a} \\
i_{b} \\
i_{c}
\end{array}\right]+p\left[\begin{array}{l}
\psi_{A} \\
\psi_{B} \\
\psi_{C} \\
\psi_{a} \\
\psi_{b} \\
\psi_{c}
\end{array}\right]
$$

where $u_{A}, u_{B}$, and $u_{C}$ are stator phase voltage, $u_{a}, u_{b}$, and $u_{c}$ are rotor phase voltage, $i_{A}, i_{B}$, and $i_{C}$ are stator phase current, $i_{a}, i_{b}$, and $i_{c}$ are rotor phase current, $\psi_{A}, \psi_{B}$, and $\psi_{C}$ are magnetic linkage of stator winding resistance, $\psi_{a}, \psi_{b}$, and $\psi_{c}$

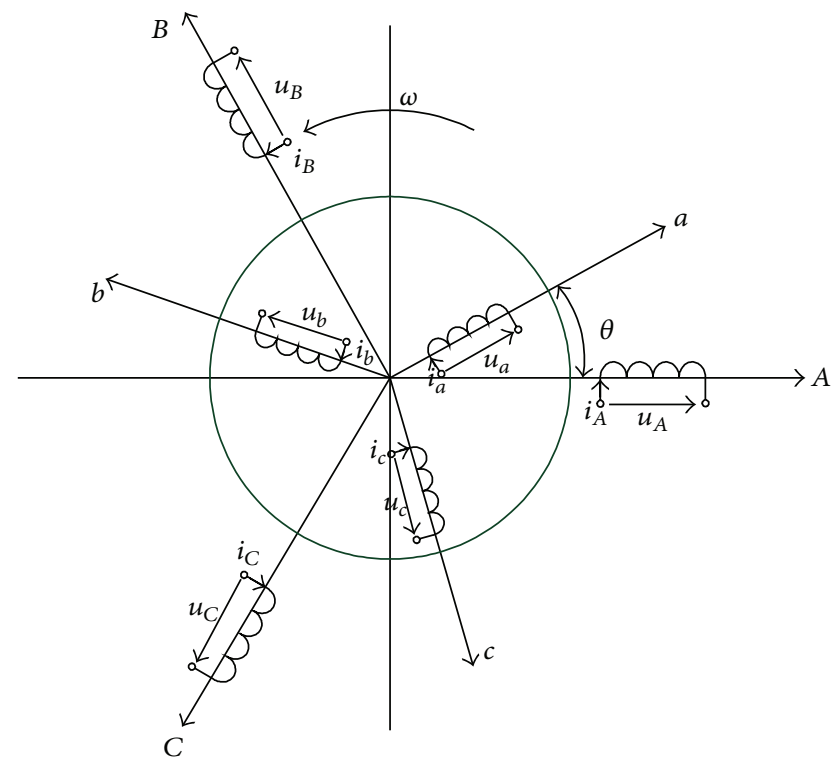

FIgure 1: The physical model of ACIM.

are magnetic linkage of rotor winding resistance, $R_{s}$ is stator resistance, $R_{r}$ is rotor resistance, and $p$ is differential operator.

Based on the principle that the magnetic linkage of every winding resistance is equal to its self-inductance magnetic linkage plus mutual inductance magnetic linkage with other winding resistances, the magnetic linkage matrix equation can be written as

$$
\left[\begin{array}{l}
\psi_{A} \\
\psi_{B} \\
\psi_{C} \\
\psi_{a} \\
\psi_{b} \\
\psi_{c}
\end{array}\right]=\left[\begin{array}{llllll}
L_{A A} & L_{A B} & L_{A C} & L_{A a} & L_{A b} & L_{A c} \\
L_{B A} & L_{B B} & L_{B C} & L_{B a} & L_{B b} & L_{B c} \\
L_{C A} & L_{C B} & L_{C C} & L_{C a} & L_{C b} & L_{C c} \\
L_{a A} & L_{a B} & L_{a C} & L_{a a} & L_{a b} & L_{a c} \\
L_{b A} & L_{b B} & L_{b C} & L_{b a} & L_{b b} & L_{b c} \\
L_{c A} & L_{c B} & L_{c C} & L_{c a} & L_{c b} & L_{c c}
\end{array}\right]\left[\begin{array}{c}
i_{A} \\
i_{B} \\
i_{C} \\
i_{a} \\
i_{b} \\
i_{c}
\end{array}\right],
$$

where $L_{A A}$ is self-inductance and $L_{A B}$ is mutual inductance.

The torque equation can be expressed as

$$
\begin{aligned}
T_{e}=n_{p} L_{m} & {\left[\left(i_{A} i_{a}+i_{B} i_{b}+i_{C} i_{c}\right) \sin \theta\right.} \\
& +\left(i_{A} i_{b}+i_{B} i_{c}+i_{C} i_{a}\right) \sin \left(\theta+120^{\circ}\right) \\
& \left.+\left(i_{A} i_{c}+i_{B} i_{a}+i_{C} i_{b}\right) \sin \left(\theta-120^{\circ}\right)\right],
\end{aligned}
$$

where $n_{p}$ is pole pairs, $L_{m}$ is mutual inductance, and $\theta$ is electrical degree difference between $a$ axis and $A$ axis.

The ACIM, whose mathematical model is very complex, is very difficult to be controlled in practical application. The vector control algorithm controls ACIM as DC motor through coordinate transformation for the problem that the mathematical model ACIM is very complex so that the governing performance of ACIM can be comparable with DC motor. 


\section{Improved Variable Gain PID Control Algorithm Design}

The fundamental thought of traditional variable gain PID control algorithm is matching the cumulative velocity of integral value with the magnitude of deviation. The integral action reduces to nothing for preventing integral saturation when system deviation is large and is reinforced for improving the stability of velocity when system deviation is small. The more desirable situation is matching the magnitude of proportional coefficient with deviation. The action of proportional part is reinforced for improving the dynamic performance of system when system deviation is large and reduces for preventing overshoot when system deviation is small. This paper designs an improved variable gain PID control algorithm based on improving the variable gain PID control algorithm.

The proportional and integral term of improved variable gain PID control algorithm can be expressed as

$$
\begin{aligned}
u(k)= & \left(k_{p}+x[e(k)]\right) e(k) \\
& +k_{i}\left\{\sum_{i=0}^{k-1} e(i)+y[e(k)] e(k)\right\} T,
\end{aligned}
$$

where $x[e(k)]$ and $y[e(k)]$ are the functions of deviation $e(k)$. As $e(k)$ increases, $x[e(k)]$ increases and $y[e(k)]$ reduces. As $e(k)$ reduces, $x[e(k)]$ reduces and $y[e(k)]$ increases.

The expression of $x[e(k)]$ can be described as

$$
\begin{aligned}
& x[e(k)] \\
& = \begin{cases}k_{p 1}^{\prime}, & |e(k)| \leq e_{2}, \\
\frac{k_{p 2}^{\prime}-k_{p 1}^{\prime}}{e_{1}}\left(|e(k)|-e_{2}\right)+k_{p 1}^{\prime}, & e_{2}<|e(k)| \leq e_{1}+e_{2}, \\
k_{p 2}^{\prime}, & |e(k)|>e_{1}+e_{2},\end{cases}
\end{aligned}
$$

where parameters $e_{1}, e_{2}, k_{p 1}^{\prime}$, and $k_{p 2}^{\prime}$ are necessary to be ensured, $0 \leq k_{p 1}^{\prime}<k_{p 2}^{\prime}$. On one hand, the chosen values of these four parameters must satisfy the condition of system stability. On the other hand, the chosen values of $e_{1}$ and $k_{p 2}^{\prime}$ must meet the condition of velocity, and the chosen values of $e_{2}$ and $k_{p 1}^{\prime}$ must meet the condition of no velocity overshoot.

The value of $x[e(k)]$ varies in the range of interval $\left[k_{p 1}^{\prime}, k_{p 2}^{\prime}\right]$

When $|e(k)|>e_{1}+e_{2}, x[e(k)]$ is equal to $k_{p 2}^{\prime}$ and the proportional coefficient of control algorithm is equal to $k_{p}+$ $k_{p 2}^{\prime}$, for improving the dynamic performance of system.

When $|e(k)| \leq e_{2}, x[e(k)]$ is equal to $k_{p 1}^{\prime}$ and the proportional coefficient of control algorithm is equal to the minimum value $k_{p}+k_{p 1}^{\prime}$, for preventing overshoot.

When $e_{2}<|e(k)| \leq e_{1}+e_{2}$, the value of $x[e(k)]$ which is in the range of interval $\left[k_{p 1}^{\prime}, k_{p 2}^{\prime}\right]$ and the proportional coefficient of control algorithm which is in the range of interval $\left[k_{p}+k_{p 1}^{\prime}, k_{p}+k_{p 2}^{\prime}\right]$ vary with the magnitude of $|e(k)|$.
The expression of $x[e(k)]$ can be described as

$$
\begin{aligned}
& y[e(k)] \\
& \quad= \begin{cases}1, & |e(k)| \leq e_{4}, \\
\frac{k_{i}^{\prime}-1}{e_{3}}\left(|e(k)|-e_{4}\right)+1, & e_{4}<|e(k)| \leq e_{3}+e_{4}, \\
k_{i}^{\prime}, & |e(k)|>e_{3}+e_{4},\end{cases}
\end{aligned}
$$

where parameters $e_{3}, e_{4}$, and $k_{i}^{\prime}$ are necessary to be ensured, $0 \leq k_{i}^{\prime}<1$. On one hand, the chosen values of these three parameters must satisfy the condition of system stability. On the other hand, the chosen values of $e_{3}$ and $k_{i}^{\prime}$ must meet the condition of no integral saturation and velocity overshoot, and the chosen value of $e_{4}$ must make velocity stability rapidly.

The value of $y[e(k)]$ varies in the range of interval $\left[k_{i}^{\prime}, 1\right]$.

When $|e(k)|>e_{3}+e_{4}$, the value of $y[e(k)]$ is equal to $k_{i}^{\prime}$, for reducing the integral action to the lowest or not accumulating the current value of $e(k)$.

When $|e(k)| \leq e_{4}$, the integral term is the same as the general, for increasing the integral action to the highest and accumulating the current value of $e(k)$.

When $e_{4}<|e(k)| \leq e_{3}+e_{4}$, the value of $y[e(k)]$, which is in the range of interval $\left[k_{i}^{\prime}, 1\right]$, varies with the magnitude of $|e(k)|$ and the integral term accumulates part current value of $e(k)$. Thus, the integral velocity is in the range of $k_{i}\left\{\sum_{i=0}^{k-1} e(i)+\right.$ $\left.k_{i}^{\prime} e(k)\right\} T$ to $k_{i} \sum_{i=0}^{k} e(i) T$.

In order to increase the regulating range of improved variable gain PID control algorithm, the values of parameters $e_{1}, e_{2}, e_{3}$, and $e_{4}$ must be decided by the maximum value of deviation after desired value varies which are not fixed values. Thus, we can get

$$
\begin{aligned}
& e_{1}=\left|e_{\max }\right| m_{1}, \\
& e_{2}=\left|e_{\max }\right| m_{2}, \\
& e_{3}=\left|e_{\max }\right| m_{3}, \\
& e_{4}=\left|e_{\max }\right| m_{4},
\end{aligned}
$$

where $e_{\max }$ is the maximum value of deviation between desired value and feedback value after the desired value of controller input changes and parameters $m_{1}, m_{2}, m_{3}$, and $m_{4}$ are necessary to be ensured, which must satisfy $0<m_{i}<1$, $i=1,2,3,4,0<m_{1}+m_{2}<1$, and $0<m_{3}+m_{4}<$ 1. First of all, the chosen values of these four parameters must satisfy the condition of system stability. Secondly, the chosen value of $m_{1}$ must make velocity stability rapidly, and the chosen value of $m_{2}$ must meet the condition of no velocity overshoot, and the chosen value of $m_{3}$ must meet the condition of no integral saturation and velocity overshoot, and the chosen value of $m_{4}$ must make velocity stability rapidly. 


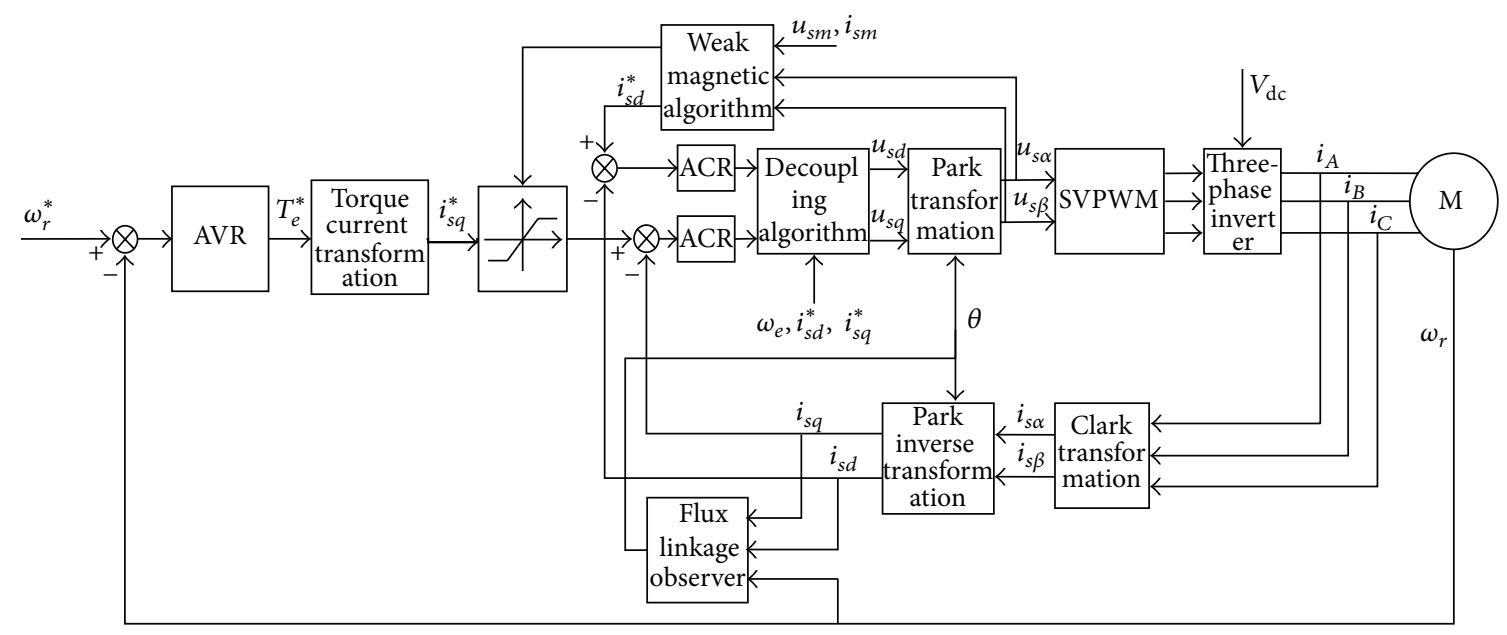

FIGURE 2: The block diagram of vector control algorithm based on improved variable gain PID controller motor.

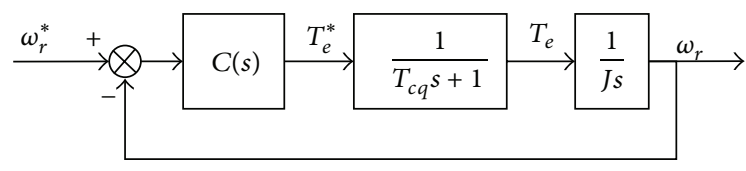

FIGURE 3: The transfer function block diagram of vector control algorithm based on improved variable gain PID controller.

Finally, the improved variable gain PID control algorithm is obtained as

$$
\begin{aligned}
u(k)= & \left(k_{p}+x[e(k)]\right) e(k) \\
& +k_{i}\left\{\sum_{i=0}^{k-1} e(i)+y[e(k)] e(k)\right\} T \\
& +k_{d} \frac{e(k)-e(k-1)}{T} .
\end{aligned}
$$

Because requirement of improved variable gain PID control algorithm to the values of parameters $m_{1}, m_{2}, m_{3}$, $m_{4}, k_{p 1}^{\prime}, k_{p 2}^{\prime}$, and $k_{i}^{\prime}$ is not accurate, the values are easy to be ensured.

\section{Vector Control Algorithm for Vehicle Asynchronous Motor Based on Improved Variable Gain PID Controller}

The block diagram of vector control algorithm for EV ACIM based on improved variable gain PID controller is obtained in Figure 2. This algorithm, which uses rotator flux oriented, uses velocity and current double closed-loop control algorithm in control structure.

In outer loop control, collect motor rotor velocity $\omega_{r}$ via revolution velocity transducer from the ACIM side. Then, set the deviation between expected rotor velocity $\omega_{r}^{*}$ and feedback rotor velocity $\omega_{r}$ as the input of automatic voltage regulator (AVR), and the output is expected electromagnetic torque $T_{e}^{*}$. The expected electromagnetic torque via torquecurrent transformation and slicing obtains the inner loop expected torque current $i_{s q}^{*}$. The requisite parameter $\theta$ of Park transformation and Park inverse transformation is provided by flux linkage observer. The inputs of weak magnetic block are $u_{s \alpha}, u_{s \beta}$, the maximum output voltage value of inverter is $u_{s m}$, and the maximum motor current value in safe running is $i_{s m}$, and the outputs are the slicing values of expected excitation current $i_{s d}^{*}$ and expected torque current $i_{s q}^{*}$.

The transfer function block diagram of vector control algorithm for EV asynchronous motor based on improved variable gain PID controller can be obtained in Figure 2.

In Figure 3, $1 / T_{c q} s+1$ is closed-loop transfer function of torque control system. So the transfer function of controlled object can be expressed as

$$
G(s)=\frac{1}{T_{c q} s+1} * \frac{1}{J s}=\frac{1}{\left(\sigma L_{s} / k_{i q}\right) s+1} * \frac{1}{J s},
$$

where $k_{i q}$ is $q$ loop integral coefficient of vector control algorithm current loop, $\sigma$ is leakage inductance coefficient of ACIM, $L_{s}$ is stator inductance of ACIM, and $J$ is moment of inertia of ACIM.

The differentiation element of PID control algorithm, which is sensitive to the noise of input signal, is not used in the system which has bigger noise in general. Thus, only PI control in the velocity loop controller of vector control algorithm for EV ACIM in general is used.

The transfer function of velocity loop controller based on improved variable gain PID control algorithm is given as

$$
C(s)=\frac{\left(k_{p}+x[e(k)]\right) s+k_{i} y[e(k)]}{s},
$$

where $k_{p}$ is proportional coefficient of traditional PID control algorithm, $k_{i}$ is integral coefficient of PID control algorithm, $k_{p 1}^{\prime} \leq x[e(k)] \leq k_{p 2}^{\prime}$, and $k_{i}^{\prime} \leq y[e(k)] \leq 1$. Here $k_{p 1}^{\prime}, k_{p 2}^{\prime}$, and $k_{i}^{\prime}$ are parameters of improved variable gain PID control algorithm to be determined. 


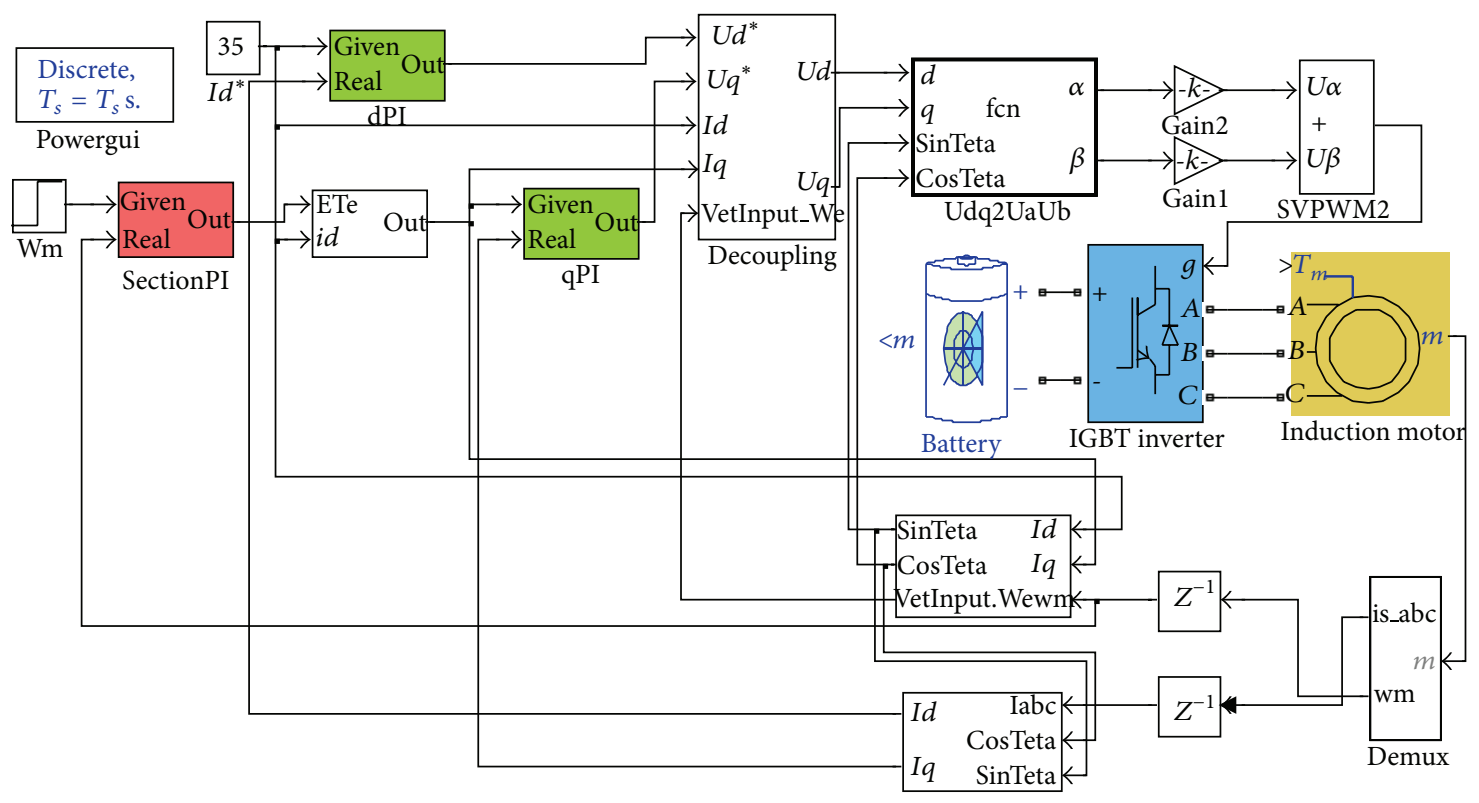

FIgURE 4: Simulation diagram constructed by Matlab/Simulink.

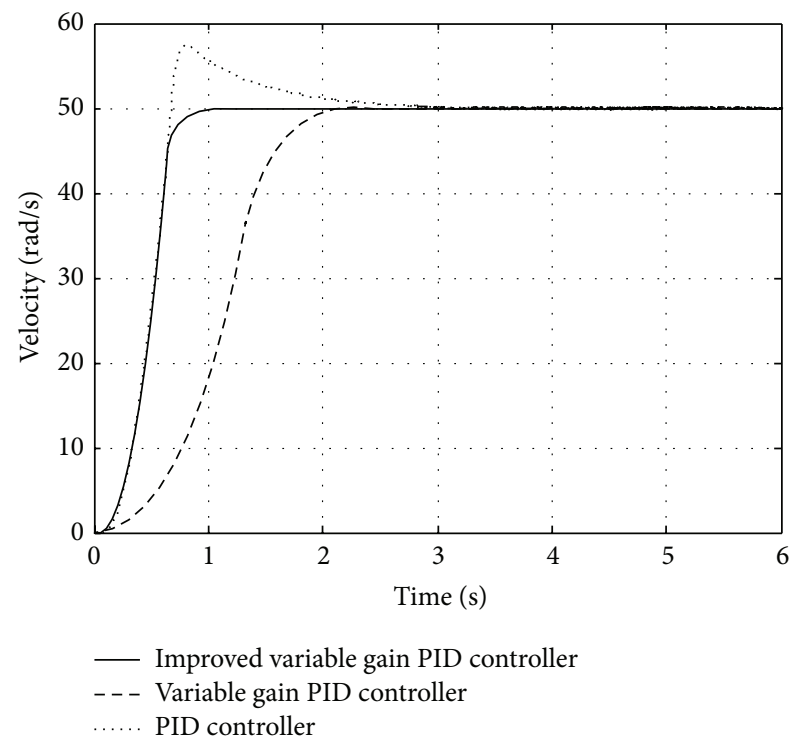

FIGURE 5: The simulation result of motor in low-velocity range.

Therefore, the closed-loop transfer function of system is obtained as

$$
\begin{aligned}
\varphi(s) & =\frac{C(s) G(s)}{1+C(s) G(s)} \\
& =\frac{\left(k_{p}+x[e(k)]\right) s+k_{i} y[e(k)]}{\left(\sigma L_{s} J / k_{i q}\right) s^{3}+J s^{2}+\left(k_{p}+x[e(k)]\right) s+k_{i} y[e(k)]} .
\end{aligned}
$$

Using Routh stability criterion [18] to judge the stability of system can obtain the stability condition of system as

$$
\begin{aligned}
& k_{p 1}^{\prime}>\frac{\sigma L_{s} k_{i}}{k_{i q}}-k_{p}, \\
& k_{i}^{\prime}>0 .
\end{aligned}
$$

The system can be stable if the values of parameters $k_{p 1}^{\prime}$ and $k_{i}^{\prime}$ satisfy the condition of (9) when designing the vector control algorithm for EV ACIM based on improved variable gain PID controller.

\section{Simulation and Interpretation of Results}

To study the improvements of the improved variable gain PID control algorithm, it is imperative to compare it to classical PID control algorithm through simulation. The motor parameters of $20 \mathrm{~kW}$ ACIM which is used in simulation are given in Table 1.

Through debugging the simulation model of specific ACIM described in Table 1, proportional and integral gains as 0.63797 and 30.158 , respectively, can be obtained, and the values of parameters of improved variable gain PID control algorithm in the velocity loop controller are $k_{p}=2.26, k_{i}=$ $3.58, m_{1}=0.06, m_{2}=m_{3}=0.04, m_{4}=0.16, k_{p 1}^{\prime}=0, k_{p 2}^{\prime}=4$, and $k_{i}^{\prime}=0.01 . k_{p 1}^{\prime}>\sigma L_{s} k_{i} / k_{i q}-k_{p}=-2.2538$ and $k_{i}^{\prime}>0$; these satisfy the stability condition of system.

As shown in Figure 3, take a PID controller for example for comparison. Sample time $T_{s}$ is $500 \mu$ s which is the sample period of the closed-loop system.

5.1. Low-Velocity Range. EV low running velocity is about $10 \mathrm{~km} / \mathrm{h}$, and the corresponding motor velocity is about 


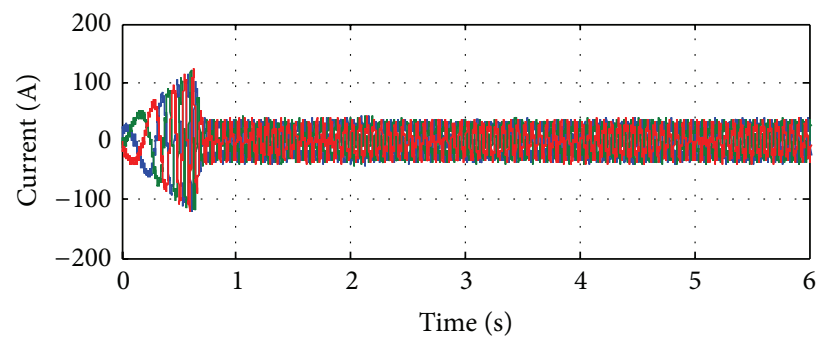

(a) Three-phase current with PID controller

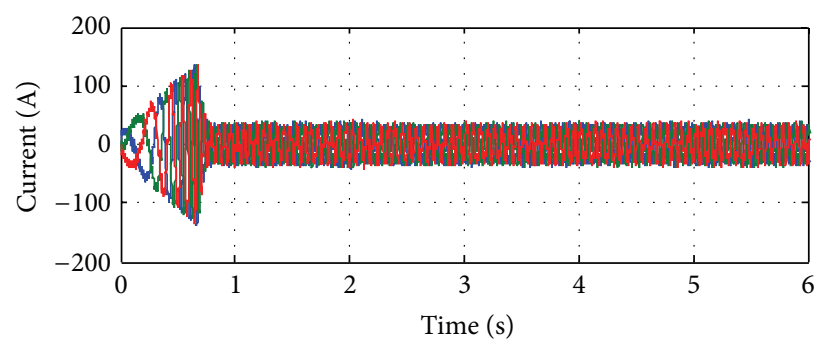

(b) Three-phase current with variable gain PID controller

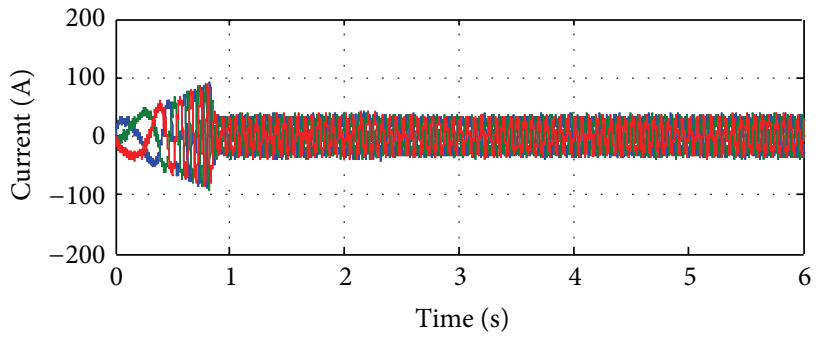

(c) Three-phase current with improved variable gain PID controller

FIGURE 6: The simulation result of three-phase current.

TABLE 1: The parameters of ACIM.

Rated line voltage $U_{N}$

Rated torque $T_{e N}$

Rated velocity $n_{N}$

Stator resistance $R_{1}$

Rotor resistance $R_{2}$

Stator leakage inductance $L_{\text {ls }}$

Rotor leakage inductance $L_{\mathrm{lr}}$

Mutual inductance $L_{m}$

Pole pairs

$50 \mathrm{rad} / \mathrm{s}$. If the expected velocity is motor nominal velocity of $50 \mathrm{rad} / \mathrm{s}$ in simulation, the control result is illustrated in Figure 5.

In Figure 5, the response time of the PID controller is about $3 \mathrm{~s}$, and the overshoot is about $14 \%$. The response time of variable gain PID controller is about $2 \mathrm{~s}$, and the overshoot is 0 . However, the proposed controller can still track the desired velocity less than $1 \mathrm{~s}$ without 0 overshoots.

In Figure 6, compared with PID controller and variable gain PID controller, the peak of three-phase current is less

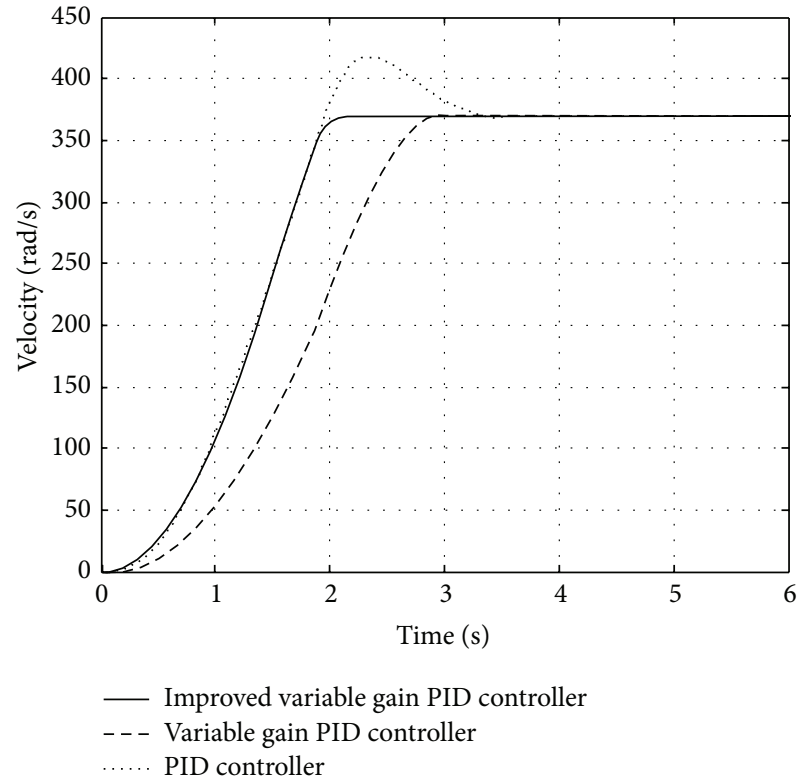

FIGURE 7: The simulation result of motor in moderate-velocity range. 


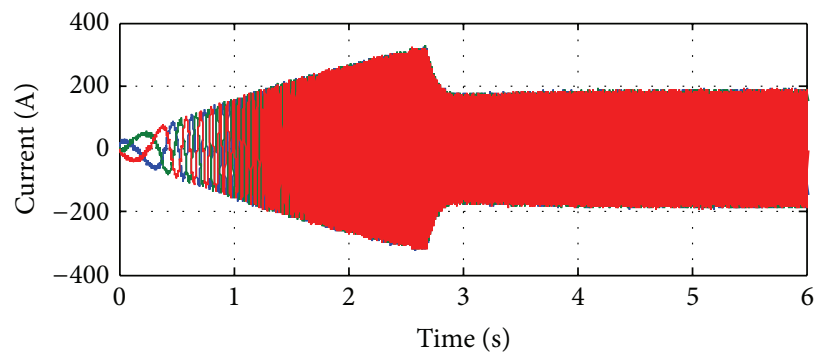

(a) Three-phase current with PID controller

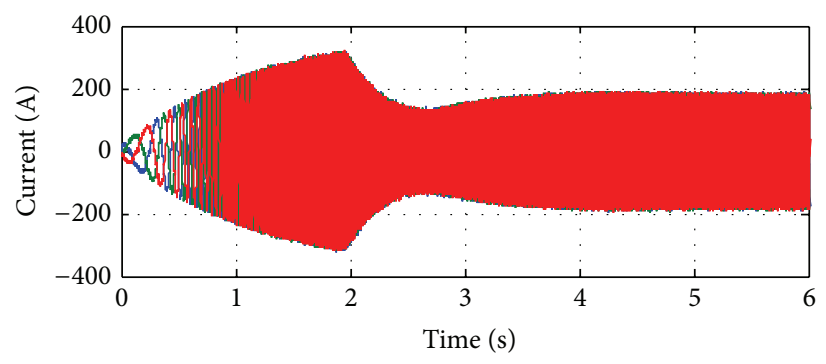

(b) Three-phase current with variable gain PID controller

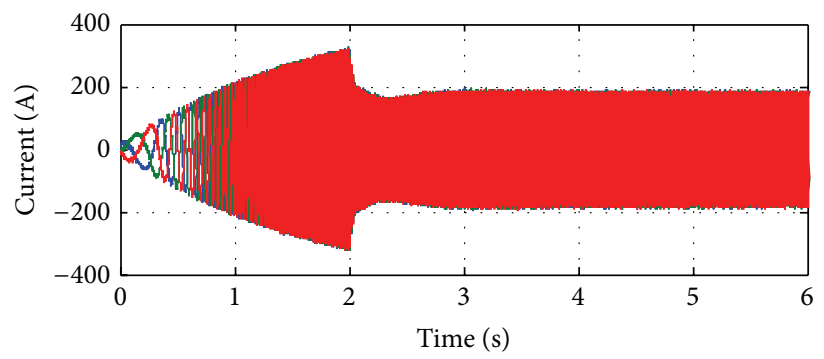

(c) Three-phase current with improved variable gain PID controller

FIGURE 8: The simulation result of three-phase current.

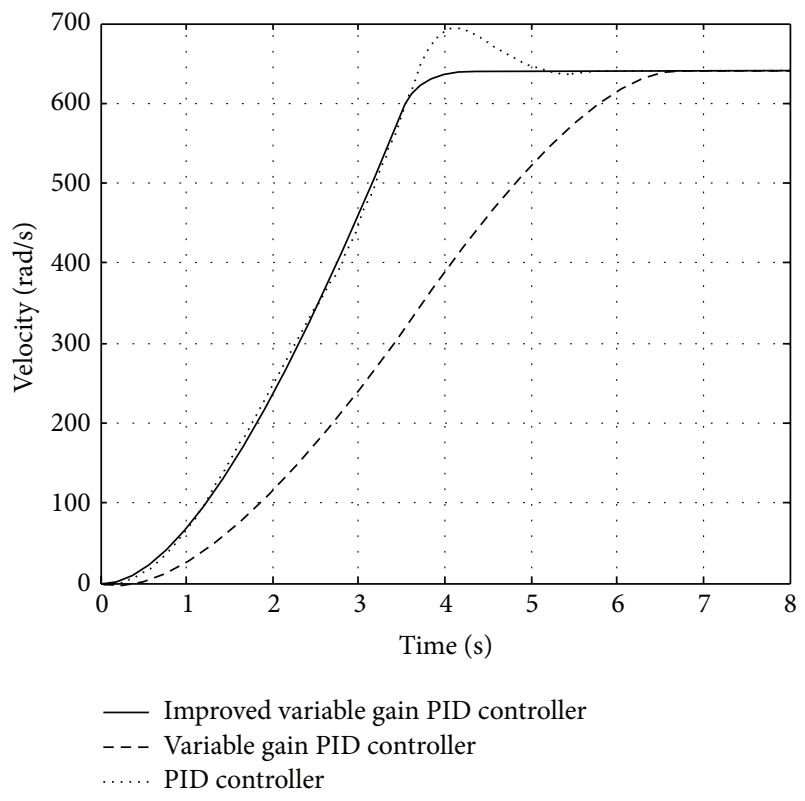

FIGURE 9: The simulation result of motor in high-velocity range. than $100 \mathrm{~A}$. In addition, it is investigated that the proposed controller is valid by comparison with the other PID controllers.

5.2. Moderate-Velocity Range. EV normal running velocity is $80 \mathrm{~km} / \mathrm{h}$ to $100 \mathrm{~km} / \mathrm{h}$, and the corresponding motor velocity is about $370 \mathrm{rad} / \mathrm{s}$. If the expected velocity is motor nominal velocity of $370 \mathrm{rad} / \mathrm{s}$ in simulation, the control result is illustrated in Figure 7.

In Figure 7, the response time of the PID controller is about $3.5 \mathrm{~s}$, and the overshoot is about $10.8 \%$. The response time of variable gain PID controller is about $3 \mathrm{~s}$, and the overshoot is 0 . However, the proposed controller can still track the desired velocity less than $2 \mathrm{~s}$ without 0 overshoots.

In Figure 8, compared with PID controller and variable gain PID controller, three-phase current changes slowly and the response time is smaller. In addition, it is investigated that the proposed controller is valid by comparison with the other PID controllers.

5.3. High-Velocity Range. EV high running velocity is about $120 \mathrm{~km} / \mathrm{h}$, and the corresponding motor velocity is about 


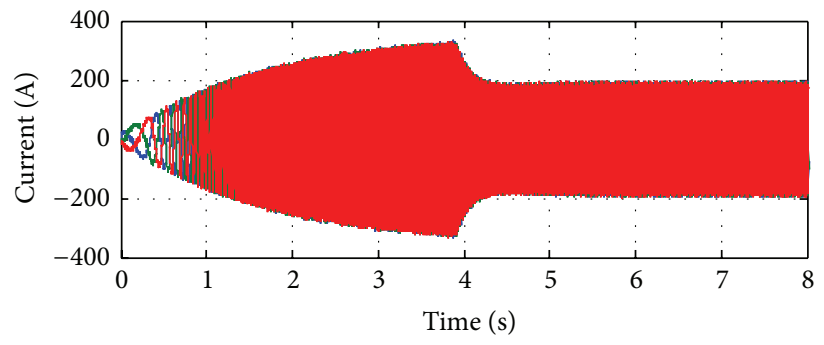

(a) Three-phase current with PID controller

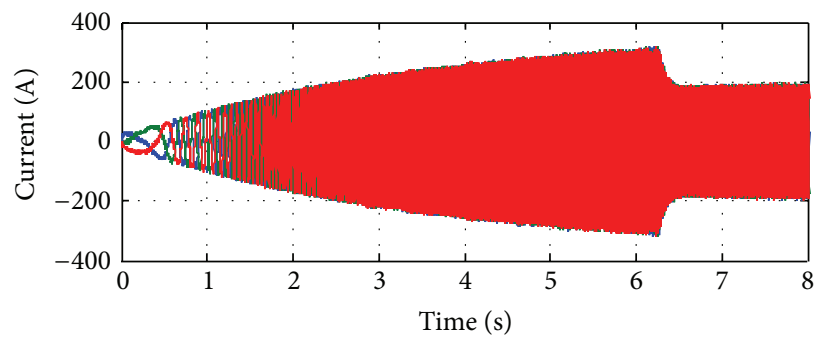

(b) Three-phase current with variable gain PID controller

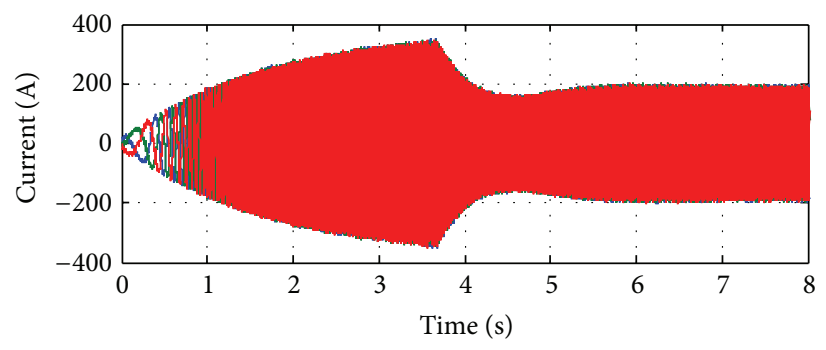

(c) Three-phase current with improved variable gain PID controller

FIGURE 10: The simulation result of three-phase current.

$630 \mathrm{rad} / \mathrm{s}$. If the given expected velocity is motor nominal velocity of $630 \mathrm{rad} / \mathrm{s}$ in simulation, the control result is illustrated in Figure 9.

In Figure 9, the response time of the PID controller is about $5 \mathrm{~s}$, and the overshoot is about $11.1 \%$. The response time of variable gain PID controller is about $7 \mathrm{~s}$, and the overshoot is 0 . However, the proposed controller can still track the desired velocity less than $4 \mathrm{~s}$ without 0 overshoots.

In Figure 10, compared with PID controller and variable gain PID controller, three-phase current changes slowly and the response time is smaller. In addition, it is investigated that the proposed controller is valid by comparison with the other PID controllers.

5.4. Variable Velocity Range. In order to test wheter the method designed in this paper can satisfy the application needs in velocity, we make simulation of motor in variable velocity range with the initial expected velocity as $630 \mathrm{rad} / \mathrm{s}$ and turn to $300 \mathrm{rad} / \mathrm{s}$ at the 4 th second. The control result is illustrated in Figure 11.

As shown in Figure 11, the response time of the PID controller is about $2.5 \mathrm{~s}$, and the overshoot is about $7.9 \%$. The response time of variable gain PID controller is about $3 \mathrm{~s}$, and

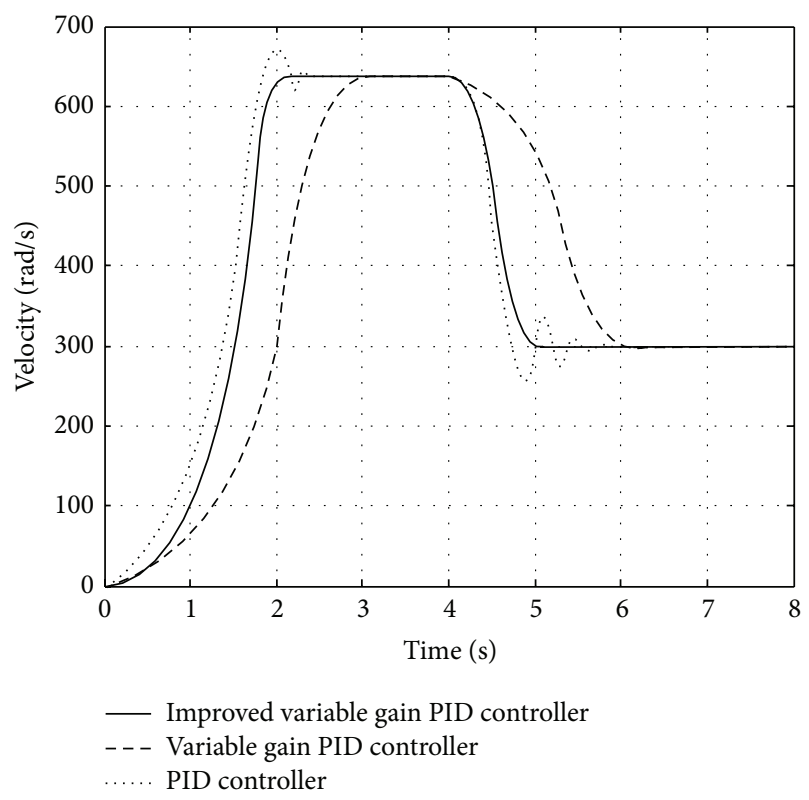

FIGURE 11: The simulation result of motor in variable velocity range. 
the overshoot is 0 . However, the proposed controller can still track the desired velocity less than $4 \mathrm{~s}$ without 0 overshoots.

\section{Conclusion}

This paper has presented a novel approach in the automotive field to implement the improved variable gain PID controller to control EV ACIM. In this paper, the design of variable gain PID controller and stability analysis have been presented along with simulation. The simulation results qualitatively demonstrate that the improved variable gain PID controller could improve on the control of motor velocity in the EV versus using the classical PID control method. In addition, this control method can satisfy the demands of EV driving system dynamic performance and acceleration performance when EV runs in low velocity, moderate velocity, high velocity, and variable velocity.

\section{Conflict of Interests}

The authors declare that there is no conflict of interests regarding the publication of this paper.

\section{References}

[1] C. Larish, D. Piyabongkarn, V. Tsourapas, and R. Rajamani, "A new predictive lateral load transfer ratio for rollover prevention systems," IEEE Transactions on Vehicular Technology, vol. 62, no. 7, pp. 2928-2936, 2013.

[2] S.-I. Sakai, H. Sado, and Y. Hori, "Motion control in an electric vehicle with four independently driven in-wheel motors," IEEE/ASME Transactions on Mechatronics, vol. 4, no. 1, pp. 9$16,1999$.

[3] Q. Li, Z. Zhang, and W. Zhao, "Dynamic control for fourwheel independent drive electric vehicle," in Proceedings of the International Conference on Computer Science and Electronics Engineering (ICCSEE '12), vol. 3, pp. 252-256, Hangzhou, China, March 2012.

[4] T. Shim and D. Margolis, "Model-based road friction estimation,” Vehicle System Dynamics, vol. 41, no. 4, pp. 249-276, 2004.

[5] D. Chabrol, C. Aussagues, and V. David, "A spatial and temporal partitioning approach for dependable automotive systems," in Proceedings of the IEEE Conference on Emerging Technologies \& Factory Automation (ETFA '09), pp. 1-8, Mallorca, Spain, September 2009.

[6] M. Stecher, N. Jensen, M. Denison et al., "Key technologies for system-integration in the automotive and industrial applications," IEEE Transactions on Power Electronics, vol. 20, no. 3, pp. 537-549, 2005.

[7] D. Zhang and L. Yu, "Exponential state estimation for Markovian jumping neural networks with time-varying discrete and distributed delays," Neural Networks, vol. 35, pp. 103-111, 2012.

[8] V. P. Petkov, G. K. Balachandran, and J. Beintner, "A fully differential charge-balanced accelerometer for electronic stability control," IEEE Journal of Solid-State Circuits, vol. 49, no. 1, pp. 262-269, 2014.

[9] C. F. Caruntu, M. Lazar, R. H. Gielen, P. P. J. van den Bosch, and S. Di Cairano, "Lyapunov based predictive control of vehicle drivetrains over CAN," Control Engineering Practice, vol. 21, no. 12, pp. 1884-1898, 2013.
[10] J. Cheng, H. Zhu, S. Zhong, F. Zheng, and Y. Zeng, "Finite-time filtering for switched linear systems with a mode-dependent average dwell time," Nonlinear Analysis: Hybrid Systems, vol. 15, pp. 145-156, 2015.

[11] M. B. G. Cloosterman, N. D. van de Wouw, M. P. M. H. Heemels, and H. Nijmeijer, "Robust stability of networked control systems with time-varying network-induced delays," in Proceedings of the 45th IEEE Conference on Decision and Control, pp. 4980-4985, December 2006.

[12] S. Olaru and S. Niculescu, "Predictive control for linear systems with delayed input subject to constraints," in Proceedings of the 17th World Congress the International Federation of Automatic Control (IFAC '08), pp. 11208-11213, Seoul, South Korea, July 2008.

[13] L. Hetel, J. Daafouz, and C. Iung, "Stabilization of arbitrary switched linear systems with unknown time-varying delays," IEEE Transactions on Automatic Control, vol. 51, no. 10, pp. 1668-1674, 2006.

[14] R. H. Gielen, S. Olaru, M. Lazar, W. P. Heemels, N. van de Wouw, and S.-I. Niculescu, "On polytopic inclusions as a modeling framework for systems with time-varying delays," Automatica, vol. 46, no. 3, pp. 615-619, 2010.

[15] X. Yang, Z. Wang, and W. Peng, "Coordinated control of AFS and DYC for vehicle handling and stability based on optimal guaranteed cost theory," Vehicle System Dynamics, vol. 47, no. 1, pp. 57-79, 2009.

[16] H. Du, N. Zhang, and G. Dong, "Stabilizing vehicle lateral dynamics with considerations of parameter uncertainties and control saturation through robust yaw control," IEEE Transactions on Vehicular Technology, vol. 59, no. 5, pp. 2593-2597, 2010.

[17] G. Zheng, J. D. Wu, M. W. Kuang, D. Zhang, and Y. Yang, "A disturbance rejection strategy for asynchronous motor of the electric vehicle in speed-open-loop operating mode," Advanced Materials Research, vol. 479-481, pp. 71-75, 2012.

[18] R. H. Gielen and M. Lazar, "Stabilization of networked control systems via non-monotone control Lyapunov functions," in Proceedings of the 48th IEEE Conference on Decision and Control Held Jointly with the 28th Chinese Control Conference (CDC/CCC '09), pp. 7942-7948, Shanghai, China, December 2009. 


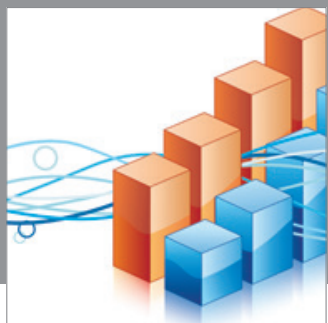

Advances in

Operations Research

mansans

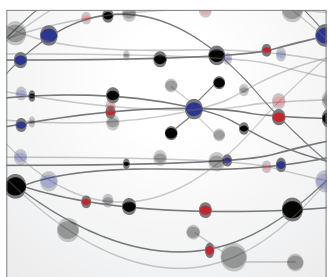

The Scientific World Journal
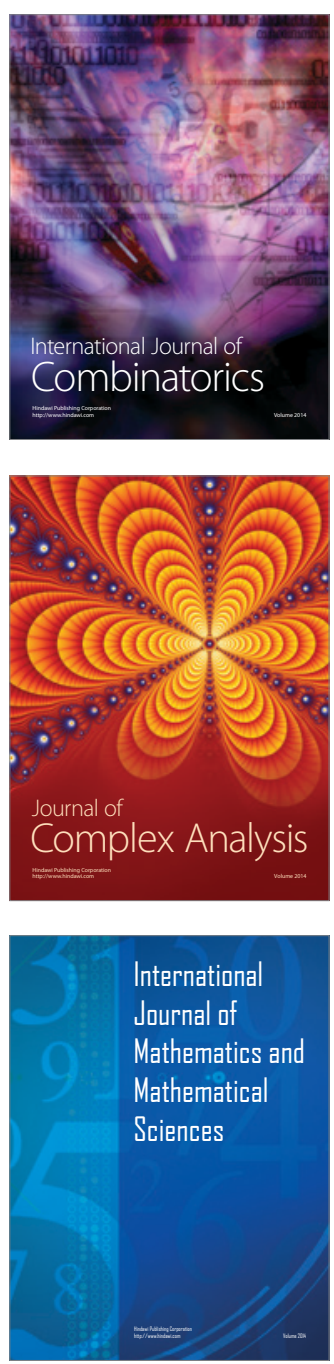
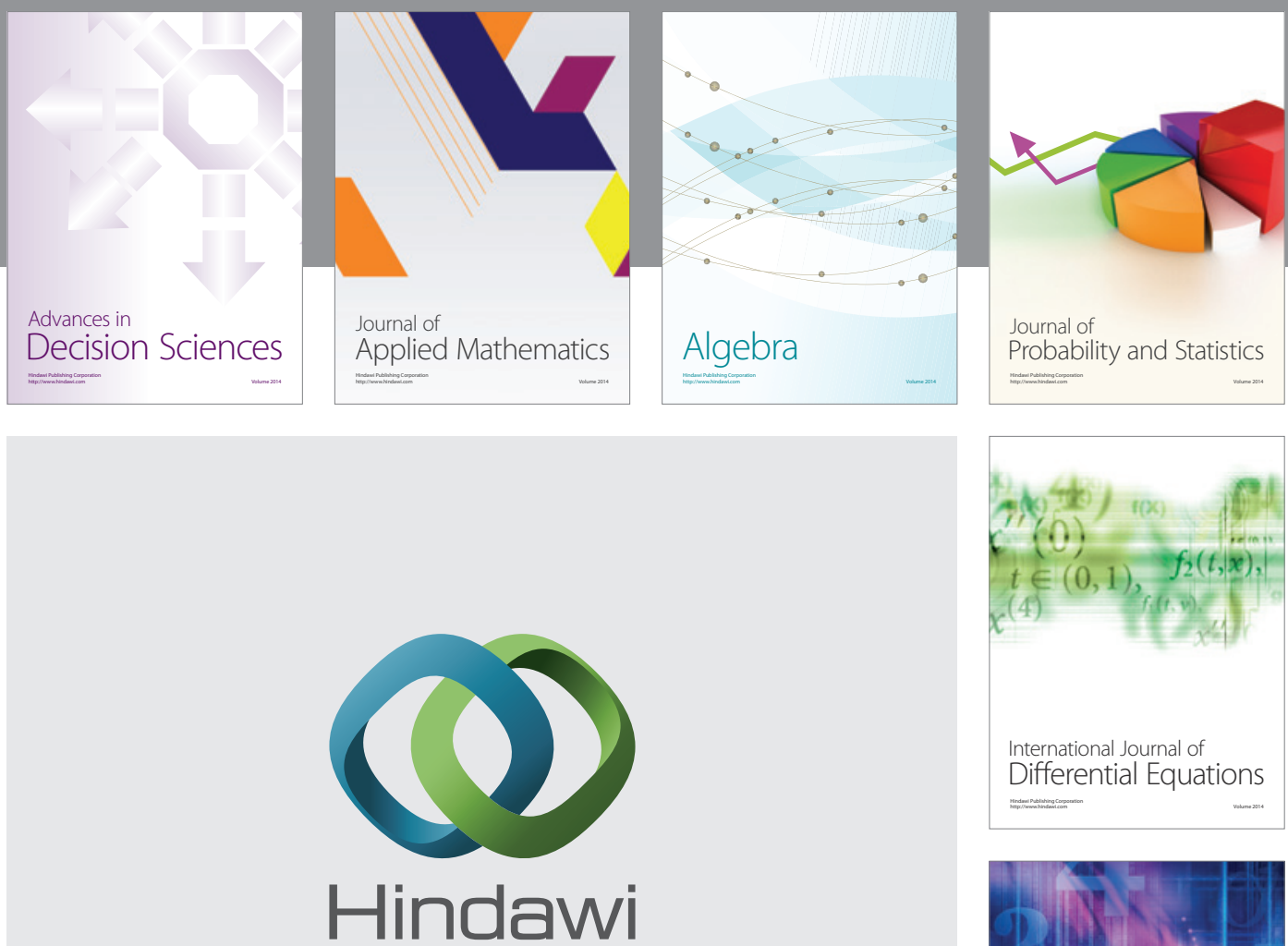

Submit your manuscripts at http://www.hindawi.com
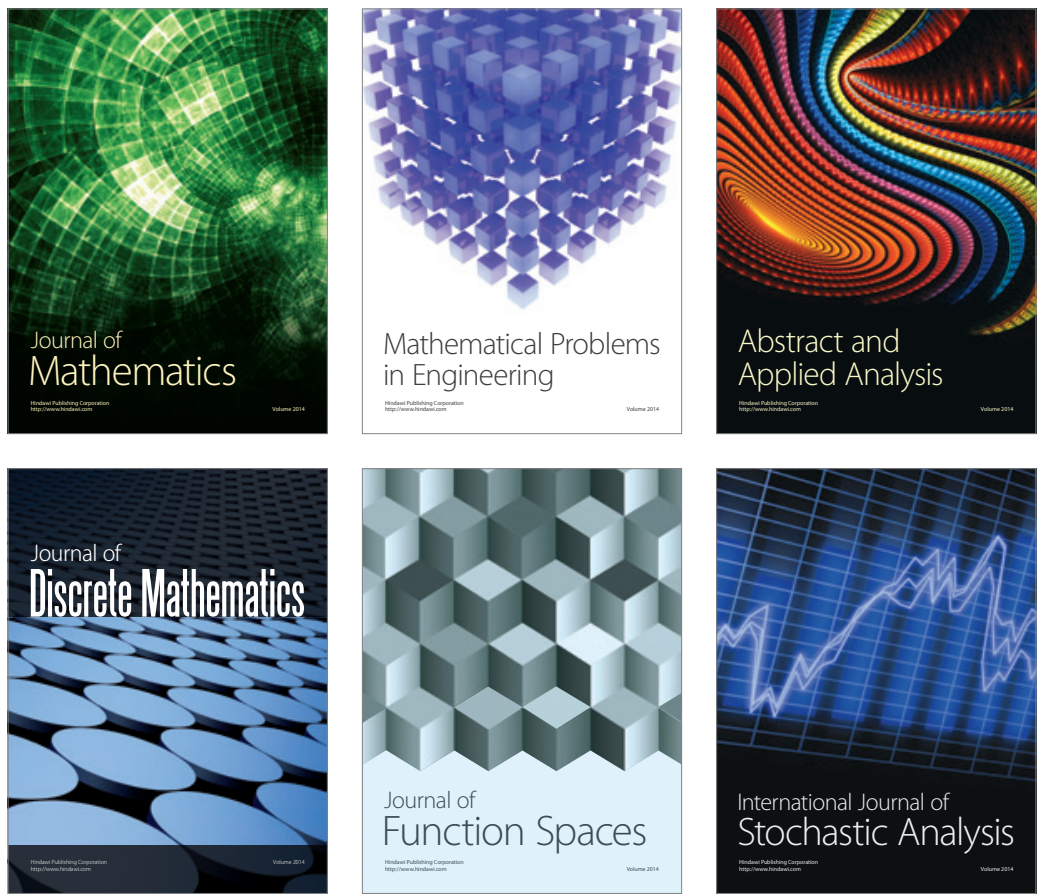

Journal of

Function Spaces

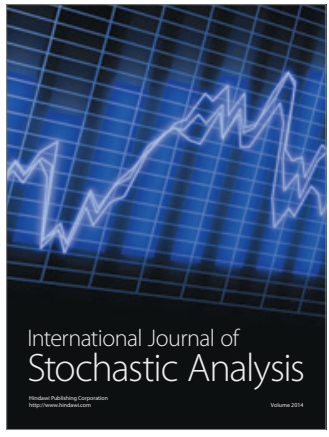

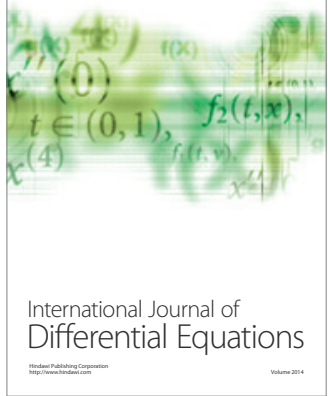
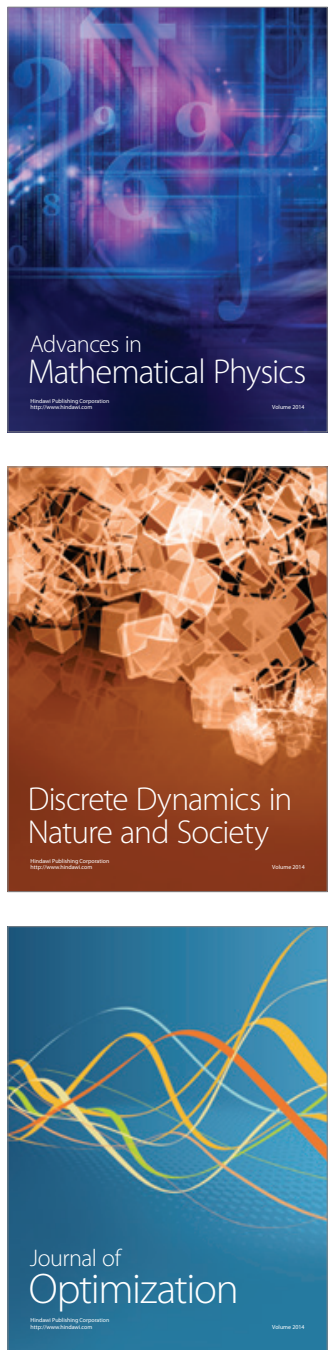